\title{
ECOLOGICAL CHARACTERISATION OF STREAMS IN TUSCANY (ITALY) FOR THE MANAGEMENT OF THE THREATENED CRAYFISH AUSTROPOTAMOBIUS PALLIPES COMPLEX
}

\author{
B. RENAI (1), S. BERTOCCHI (1), S. BRUSCONI (1), F. GHERARDI (1), F. GRANDJEAN
} (2), M. LEBBORONI (1), B. PARINET (3), C. SOUTY GROSSET (2), M.C. TROUILHE (2)

(1) Dipartimento di Biologia Animale e Genetica "Leo Pardi", Università di Firenze, Via Romana 17, 50125 Firenze (Italy).

(2) Laboratoire de Génétique et Biologie des Populations de Crustacés, Université de Poitiers, UMR-CNRS 6556, 40, avenue du Recteur Pineau, F-86022 Poitiers cedex (France).

(3) Laboratoire de Chimie de l'Eau et de l'Environnement, École Supérieure d'Ingénieurs de Poitiers, UMR-CNRS 6008, 40, avenue du Recteur Pineau, F-86022 Poitiers cedex (France).

Reçu le 16 juin 2005

Accepté le 12 janvier 2006

Received June 16, 2005 Accepted January 12, 2006

\begin{abstract}
Any initiative aimed at the management of a threatened species needs a good knowledge of its environmental requirements. Aims of this study were to individuate suitable areas for the reintroduction of crayfish species belonging to the Austropotamobius pallipes complex and to evaluate the causes of the local extinction in Tuscany (Italy) of some populations. Between May 2003 and September 2004, we collected ecological data from 19 streams of 4 catchments, 9 watercourses where crayfish were present $(\mathrm{WI})$ and 10 where they were present in the recent past and absent now (WO), and we compared them. Multivariate analyses were performed using chemico-physical and biotic parameters to examine the relationships between their values and the presence of crayfish. The results did not allow us to find significant differences between the two categories of streams, suggesting their suitability for crayfish reintroduction.
\end{abstract}

Key-words: Austropotamobius pallipes complex, Extended Biotic Index (IBE), Fluvial Functionality Index (IFF), biotic and abiotic parameters.

\section{CARACTÉRISATION ÉCOLOGIQUE DE RUISSEAUX TOSCANS (ITALIE) POUR LA GESTION DE L'ÉCREVISSE MENACÉE AUSTROPOTAMOBIUS PALLIPES COMPLEX}

\section{RÉSUMÉ}

Toute initiative de gestion d'espèces menacées nécessite une bonne connaissance de leurs exigences environnementales. Les objectifs de cette étude étaient de localiser des zones favorables à la ré-introduction d'écrevisses appartenant au complexe Austropotamobius pallipes et de déterminer les causes de l'extinction locale de certaines populations en Toscane (Italie). De mai 2003 à septembre 2004, nous avons mesuré des paramètres biotiques et abiotiques sur 19 ruisseaux, répartis dans 4 bassins 
hydrographiques différents, qui abritent actuellement des écrevisses (WI) ou qui en sont dépourvus depuis peu (WO). Des analyses multivariées ont été réalisées en prenant en compte à la fois les paramètres physico-chimiques et biotiques afin d'examiner les relations entre ces données et la présence du complexe Austropotamobius pallipes. Les résultats ne montrent pas de différence significative entre les deux catégories de ruisseaux ce qui traduirait l'aptitude des sites WO à accueillir des écrevisses.

Mots-clés : Complexe Austropotamobius pallipes, Indice biotique (IBE), Indice de Fonctionnement des Fleuves (IFF), paramètres biotiques et abiotiques.

\section{INTRODUCTION}

In Europe, the fragmented distribution of indigenous crayfish, such as the Austropotamobius pallipes complex, has been often explained as the direct and indirect result of human activities (GHERARDI and HOLDICH, 1999; SKURDAL and TAUGBOL, 2001; FÜREDER et al., 2002). These species now occur mainly in cold headwaters of good quality with high dissolved oxygen levels. As a result of this alarming situation, $A$. pallipes is included in the Red List of Threatened Animals of the International Union for the Conservation of Nature (IUCN) as a vulnerable species (IUCN, 1996), and in Annexes II and IV of European Community Directives for the Conservation of Natural Habitats and Wild Flora and Fauna (92/43/EEC and 97/62/EU) as a species requiring special conservation measures.

In recent years, plans of action (e.g. habitat conservation with the control of the spread of alien species and the restoration of indigenous populations through reintroduction programmes) have been undertaken to conserve threatened populations of crayfish in most of the European countries (BERNARDO et al., 1997; DIÉGUEZURIBEONDO et al., 1997; HOLDICH and ROGERS, 1997; REYNOLDS, 1997). A growing interest in restoring the lost crayfish production has emerged probably because stream ecologists and managers have been becoming aware of the importance of crayfish for stream systems. However, as stated by RALLO and GARCìA-ARBERAS (2002), it seems unlikely that $A$. pallipes will be ever restored to its former range or abundance because habitats have changed and even the maintenance of populations in their present locations is a difficult task in view of continuous modifications (WESTMAN, 1985).

Therefore, given the current status of $A$. pallipes complex in Italy, the assessment of environments suitable for its survival is extremely important to assure the maintenance of this threatened crayfish and to develop appropriate conservation protocols for its management. In fact although several studies on the distribution of $A$. pallipes complex have been carried out (e.g in Britain, THOMAS and INGLE, 1971; HOLDICH et al., 1978; LILLEY et al., 1979; JAY and HOLDICH, 1981; HOGGER, 1984; GODDARD and HOGGER, 1986; ROSCOE, 1986; HOLDICH and REEVE, 1987, 1991; FOSTER, 1991), only recently few analyses were attempted to identify and quantify ecological factors influencing its distribution and abundance.

It is well documented that both biotic features (e.g. bank structure and habitat characteristics) and abiotic parameters (LODGE and HILL, 1994) are of vital importance in determining the presence and the density of adult and young crayfish (HUXLEY, 1879; FOSTER, 1995; REYNOLDS and MATTHEWS, 1995; ROGERS and HOLDICH, 1995; SMITH et al., 1996; FÜREDER et al., 2002; DEMERS et al., 2003; DISTEFANO et al., 2003). BOHL (1987) and SMITH et al. (1996) observed that the fibrous and highly ramified nature of the tree roots provided a good shelter from predators, an excellent protection in times of high stream flow and it acts as debris-traps retaining leaf litter. Allochthonous leaves are primary food for crayfish (e.g. A. pallipes; REYNOLDS, 1979) in many lotic systems (MOMOT, 1984; KAUSHIK and BIRD, 1987; GODDARD, 1988), and they offer good 
shelter, particularly for small individuals (DEMERS et al., 2003). Also the exposed boulders, boulder/cobble banks and the number of riffles have a positive association with $A$. pallipes presence (NAURA and ROBINSON, 1998). The first two features reflect the importance of availability of shelter of the correct size whereas the crayfish likely exploit the riffles for feeding. The same authors (NAURA and ROBINSON, 1998) found that the canopy cover and overhanging boughs provided both shading. Moreover the invertebrates falling from the overhanging leaves into the water form a substantial part of the diet of adult and juveniles crayfish (MASON and MACDONALD, 1982; GODDARD, 1988).

Erosion is a parameter exerting a negative impact on $A$. pallipes survival (NAURA and ROBINSON, 1998) by causing loss or reduction of the amount of habitat available for crayfish. Its effect is direct by exerting a relatively high energy and indirect by increasing the concentration of suspended solids and silt in the water and on the river bed (HOGGER, 1988; SUMMERS, 1996). Several studies showed that the substratum is the most important variable related to the presence and total abundance of crayfish (FLINT and GOLDMAN, 1977; KIRJAVAINEN and WESTMAN, 1999).

Relationships between water chemistry and freshwater fauna are well known for aquatic vertebrates, but not for invertebrates (LIGHT et al., 1995). MATTHEWS and REYNOLDS (1995) maintain that changes in the chemical and thermic composition of the water (acidification, eutrophication, pollution arising from nutrient enrichment from agricultural runoff, discharges from wastewater treatment plants ecc.) are the main causes of crayfish extinction. In fact, as fishes and amphibians (MARCO et al., 1999), crayfish seem to be sensitive to nitrite concentration (as stated previously by LIU et al., 1995; ROUSE et al., 1995) that with total phosphate are reliable indicators of eutrophication. Others parameters influencing negatively the crayfish survival are ammonium (it is toxic at high concentrations), and silicate (an indicator of runoff from construction, e.g. of a new road, and of acidification in the catchment; RALLO and GARCİA-ARBERAS, 2002).

In contrast, calcium concentration is an essential component of the crayfish integument and it is indispensable for their growth and moulting (JUSSILA et al., 1995).

Finally, reintroductions must be conducted with extreme caution, since recent genetic studies showed a complex and articulated phylogeography of $A$. pallipes complex in Italy and suggested the potential for evolutionary independent lineages requiring independent conservation plans (FRATINI et al., 2004). The results confirmed the presence in the Italian peninsula of both $A$. pallipes, confined to the North-West, and of $A$. italicus, distributed across the peninsula and differentiated in four $A$. italicus subspecies, distributed in well defined geographic zones: A. i. italicus, A. i. carinthiacus, A. i. meridionalis, and A. $i$. carsicus (FRATINI et al., 2004).

Here we conducted a study in some Tuscan catchments to determine the principal abiotic and biotic characteristics of some watercourses in order to detect suitable areas for reintroduction of the $A$. pallipes complex and eventually to identify the probable causes of its local extinction. In this study, abiotic parameters were integrated with ecological features expressed also by means of standardised indices (IBE and IFF). Changes in the indices scores are widely considered as the first signals of a deterioration of the habitat and the bioindicators can be used also for diagnosing the possible causes of environmental suffering (DALE and BEYELER, 2001).

\section{MATERIAL AND METHODS}

\section{Study areas}

We assessed the distribution of $A$. pallipes complex in several areas of Tuscany. A preliminary picture of the presence of these species was obtained from previous surveys 
carried out in these years by our working team and information received from the literature, interviews, and questionnaires filled in by fishermen during Spring 2003. Then, an on-thespot investigation was carried out in Casentino (Arezzo), Lunigiana (Massa), Garfagnana (Lucca), Pistoia, and Mugello (Firenze) to verify the distribution of $A$. pallipes complex. The presence of crayfish was verified, first, by investigation during the day (1 hour manual survey) and, second, by night searching (1 hour) during the period of the species' maximum activity (summer). Several cylindrical traps $(80 \mathrm{~cm}$ long, $28 \mathrm{~cm}$ in diameter, and $4 \mathrm{~mm}$ in mesh size) baited with cat food were set in favourable spots at dusk and recovered the subsequent morning. Crayfish were recorded as absent only when they were not found after detailed searches using trapping and stone turning. At the end of this survey, four streams belonging to the Arno, Magra, and Sieve catchments were selected in Casentino, Lunigiana, and Mugello. Two of them harboured a healthy population of A. pallipes complex (WI), while in the remaining two, crayfish were present in the past (1-10 years ago) and absent now (WO). Seven watercourses ( $3 \mathrm{Wl}$ and $4 \mathrm{WO}$ ) belonging to the Serchio basin are investigated in Pistoia and Garfagnana regions (Figure 1). All the selected reaches are classified as "salmonid waters" by local Fish and Wildlife Offices and they are rather isolated from inhabited places. Their courses, running through mountainous or hilly areas, are bordered by a wooded belt of broadleaved or coniferous trees, 5-50 m wide. Only T. Carza (Mugello) and T. Acqua Bianca (Lucca) are surrounded by non intensive cultivation of fodder crops and cereals.

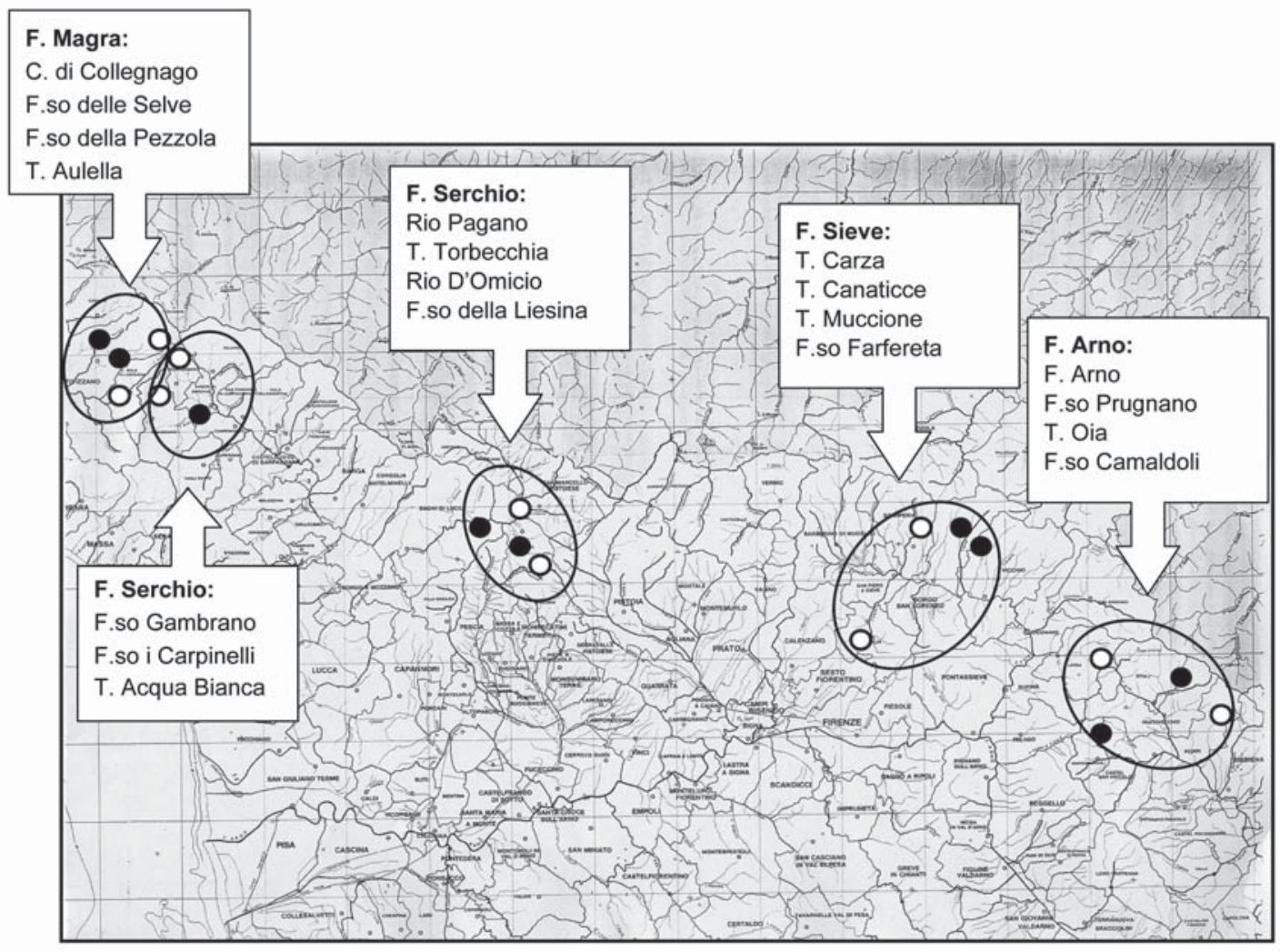

\section{Figure 1}

The study catchments and location of the watercourses with (๑) and without crayfish (O).

\section{Figure 1}

Bassins hydrographiques étudiés et localisation des ruisseaux avec $(\bullet)$ et sans (O) écrevisses. 


\section{Description of the catchments}

\section{Arno basin}

The tributaries belonging to the Arno basin (including the streams in this study) are intermittent, with a mean flow rate of about $30 \mathrm{~m}^{3} / \mathrm{sec}$. The geological formations are mostly impermeable and they are formed by clay, marl, clayed schist, limestone of marl, and sandstone. The permeable area of the catchment is not above $5 \%$ of the total. Moreover, the rocks of this basin are easily eroded.

\section{Magra basin}

Lunigiana area has a rich drainage system included in Magra basin with torrential features. The Magra is the only watercourse with characteristcs of stream. The mean flow rate is about $40 \mathrm{~m}^{3} / \mathrm{sec}$, ranging from $87 \mathrm{~m}^{3} / \mathrm{sec}$ in November to the minimum of $7.3 \mathrm{~m}^{3}$ / $\mathrm{sec}$ in August. The flow regime of these watercourses is determined by the rainfalls, which are abundant in Lunigiana, by the underlying geology and the topography. The geology of this catchment is very complex, with sandstones, serpentine rocks, jaspers, limestones, and marlclays.

\section{Serchio basin}

The watercourses in the province of Lucca are generally short, with steep slopes which give them a flow regime that responds very rapidly to rainfall. This basin has the highest annual rainfall in Italy (about $1800 \mathrm{~mm} /$ year).

Moreover, the geology features of calcareous rocks contribute to the base flow in the streams, via springs and other seepages, maintaining a reasonable flow during the summer. The mean flow rate is $46 \mathrm{~m}^{3} / \mathrm{sec}$, with the minimum value of about $6.5 \mathrm{~m}^{3} / \mathrm{sec}$. This combination of high rainfalls and groundwater means the surface water has low turbidity and there is a low level of pollution.

\section{Sieve basin}

Sieve is a right bank tributary of the Arno catchment. All the tributaries of the Sieve basin show torrential features, with a rapid increase during spates and subsequent slower phases with return to the base flow. There is a marked difference between the maximum and the minimum flow values (over $900 \mathrm{~m}^{3} / \mathrm{sec}$ in November and $0.120 \mathrm{~m}^{3} / \mathrm{sec}$ in AugustSeptember). The Sieve basin is predominantly formed by limestone and marl dated back to Miocene "Macigno del Mugello". These rocks are covered by impermeable lacustrine and fluvio-lacustrine deposits, so in summer there is little contribution to the flow from groundwater.

\section{Abiotic and biotic analyses}

A survey of physico-chemical parameters and biotic factors of habitats was carried out from May 2003 to September 2004 using the experimental protocol as follows.

The streams were divided into two categories: 9 watercourses $\mathrm{WI}$ and the remaining 10 WO. Two transects of $10 \mathrm{~m}$ each were chosen in both brooks, so that all different habitats (riffles, pools, ecc.) were analysed. For each transect, the following sets of ecological variables were recorded.

\section{Physico-chemical water parameters}

Conductivity $\left(\mu \mathrm{Scm}^{-1}\right), \mathrm{pH}$, dissolved oxygen concentration $\left(\mathrm{mgl}^{-1}\right)$, water temperature $\left({ }^{\circ} \mathrm{C}\right)$ (instantaneous, maximum and minimum) were measured (three times per transect) using a digital instrument (Multi 340i WTW) with the appropriate probes. 
Water speed $(\mathrm{m} / \mathrm{sec})$ was taken (5 measures per transect) with a hydrometric meter (ME 4001) and the maximum depth was measured with a metre stick at the beginning, middle, and end of each transect. Water speed was measured by positioning the hydrometric meter at $10 \mathrm{~cm}$ from the bottom, so that different micro-habitats were covered, including the spots where crayfish are to be found. Water samples (1 plastic bottle of $500 \mathrm{ml}$ per transect) were taken for laboratory analyses of calcium, nitrate, nitrite, ammonium, silicate and phosphate concentration using colorimetric methods (Aquamerk®, Darmstadt, Germany).

The measurements of calcium, nitrate, nitrite, ammonium, silicate and phosphate were performed on unfiltered samples. All physico-chemical parameters were measured twice seasonally so that yearly temporal variations were recorded.

\section{Basin and riparian descriptors}

During the summer, when A. pallipes complex reaches its peak of activity, we measured the following parameters:

Canopy cover. Three photos were taken for every transect using a reflex camera (35 mm objective). Values of this parameter were drawn using Image 1.32 program (Wayne Rasband, National Institute of Health, USA).

Channel substratum (proportion of bed) and availability of vegetal matter. A metal frame was used to enclose an area of $1 \times 1 \mathrm{~m}$ divided into 16 squares. The frame was launched 5 times for each transect. Inside every square, we visually estimated the percentage of surface area covered by: silt, sand ( $<2 \mathrm{~mm}$ diameter), gravel (2-64 mm), cobble (65-256 mm), boulder (> $256 \mathrm{~mm}$ ), bedrock (fixed rock), and occurrence of organic debris. When possible, organic debris was subdivided into periphyton, leaves, moss, and wood.

Shelter availability. The number of natural crevices that crayfish could use as shelters and the length of the banks $(\mathrm{cm})$ covered by organic debris, mud, roots, and rocks were quantified ( 5 segments of $1 \mathrm{~m}$ along the banks at each transect).

Bank structure. For each bank, percentages of mud, clay, roots, and rocks were calculated.

\section{Biological water quality}

The Italian Extended Biotic Index (IBE; GHETTI, 1997), based on the occurrence of macroinvertebrates, was used to assess water quality. This index reflects water quality on a scale of I to V, where I indicates optimal water quality and V polluted watercourses.

\section{Fluvial Functionality Index (IFF)}

In recent years, the assessment of Fluvial Functionality has assumed an increasing importance (ANPA, 2003). IFF derives from Riparian Channel and Environmental Inventory (RCE-I) (PETERSEN, 1992), which was designed primarily for the assessment of agricultural landscapes by scoring 16 characteristics of small stream channels.

This index integrates the results of analyses carried out on a "micro" level (e.g. IBE) with "macro" level information (e.g. land use). In this way, the fluvial environment is appraised at a wider scale. A report card is compiled with the general descriptors of the study environment (name of the stream, name of the surveyed sites, altitude, width of the river bed, etc.), followed by 14 notes about the main ecological features of the watercourse. This provides information on different environmental aspects: 1) the land use and the riparian vegetation formations; 2) the morphology and physical parameters of the bank; 3) the structure of the riverbed; and 4) biological features. The 
interpretation of these parameters by means of the principles of river ecology permits the determination of the level of deviation from the condition of maximum functionality. The value of IFF ranging from I (highest river functionality) to $\mathrm{V}$ (lowest river functionality) is obtained by adding the partial scores of every note. In the present study the Fluvial Functionality Index was calculated along a reach of $150 \mathrm{~m}$ per watercourse.

\section{Statistic analysis}

Multivariate analyses were applied using Principal Components Analysis (PCA) to discriminate WI from WO sites. The analyses were carried out using the JMP 3.1.6.1 program. The $\mathrm{G}$ test was used to compare watercourses.

\section{RESULTS}

Physical parameters are summarised in Table I. Maximum mean values of depth $(45.25 \pm 14.21 \mathrm{~cm})$ and water speed $(0.82 \pm 0.22 \mathrm{~m} / \mathrm{sec})$ were recorded during winter months, whereas the minimum mean data were measured in summer (depth: $26.35 \pm 7.00 \mathrm{~cm}$ and water speed: $0.30 \pm 0.23 \mathrm{~m} / \mathrm{sec}$ ). The water supply could vary from near desiccation to flood, with current speeds ranging from 0.01 to $1.17 \mathrm{~m} / \mathrm{sec}$. The maximum water temperature of about $20^{\circ} \mathrm{C}$ was recorded in the end of July, but values as high as those reported by LAURENT $(1988)$ in Spain $\left(23^{\circ} \mathrm{C}\right)$ were never reached. Chemical data are shown in Table II. pH values were around 8 , while conductivity ranged from 104.58 to $628.25 \mu \mathrm{Scm}^{-1}$. Dissolved oxygen concentration ranged from $6.57 \mathrm{mgl}^{-1}$ in summer to $12.21 \mathrm{mgl}^{-1}$ in winter and the minimum value was recorded in August, when the water depth was lowest and the temperature was higher than in other months. The ammonium and phosphate concentrations are not shown in the Table because their values were always below the limits of detection.

Overall, the values of the physico-chemical variables recorded in each Tuscan watercourse were in the range reported for natural and/or unpolluted streams.

The values of IFF were close to the condition of maximum functionality ranging from I (very high) to III (moderate). IBE identified the watercourses as being of good quality, ranging from I to II. The mean number of taxa (Plecoptera, Ephemeroptera, and Trichoptera families), which are the most sensitive to polluting substances, did not significantly differ between watercourses with and without crayfish $(G=0.125, \mathrm{df}=1, \mathrm{P}>0.1)$.

The Principal Components Analysis (PCA) was used to verify whether the interaction among some parameters permitted the separation of the two categories. Seven chemical parameters $(\mathrm{pH}$, conductivity, dissolved oxygen concentration, calcium, nitrate, nitrite and silicate concentration) and 5 physical parameters (instantaneous, maximum and minimum water temperatures, maximum depth, and water speed) were used.

The PCA using yearly mean values for 12 parameters did not separate WI and WO watercourses (Figure 2). The first two principal components represented up to $61.93 \%$ of the total variance $\left(\mathrm{PC}_{1}=40.62 \%, \mathrm{PC}_{2}=21.31 \%\right)$. The same result was obtained with means for every season (Spring: $\mathrm{PC}_{1}=39.60 \%, \mathrm{PC}_{2}=16.38 \%$; Summer: $\mathrm{PC}_{1}=29.73 \%$, $\mathrm{PC}_{2}=27.48 \%$; Autumn: $\quad \mathrm{PC}_{1}=43.84 \% ; \quad \mathrm{PC}_{2}=18.98 \%$; Winter: $\quad \mathrm{PC}_{1}=36.49 \%$; $\left.\mathrm{PC}_{2}=22.52 \%\right)$ and with physico-chemical parameters in summer integrated with biotic, IBE and IFF values $\left(\mathrm{PC}_{1}=17.50 \% ; \mathrm{PC}_{2}=14.93 \%\right)$ (Figure 3).

In none of the six cases a significant difference in the mean values of $\mathrm{PC}_{1}$ and $\mathrm{PC}_{2}$ between streams WI and WO was found (Table III). 


\section{Table I}

Yearly mean values ( \pm standard deviation) and ranges of physical parameters measured on every site. WI and WO denote the watercourses with and without A. pallipes complex.

\section{Tableau I}

Valeurs moyennes annuelles ( \pm écart-type) et gammes de valeurs des paramètres physiques mesurés sur chaque site. WI et WO désignent respectivement les cours d'eau avec et sans le complexe A. pallipes.

\begin{tabular}{|c|c|c|c|c|c|c|c|}
\hline & & & Depth (cm) & $\mathrm{T}\left({ }^{\circ} \mathrm{C}\right)$ & $\mathrm{T} \max \left({ }^{\circ} \mathrm{C}\right)$ & $\mathrm{T} \min \left({ }^{\circ} \mathrm{C}\right)$ & $\begin{array}{c}\text { Speed } \\
(\mathrm{m} / \mathrm{sec})\end{array}$ \\
\hline \multirow{8}{*}{ 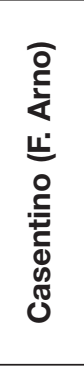 } & \multirow{2}{*}{ wo } & \multirow{2}{*}{$\begin{array}{l}\text { F. so } \\
\text { Camaldoli }\end{array}$} & $36.51 \pm 2.34$ & $8.29 \pm 0.59$ & $9.93 \pm 1.20$ & $7.09 \pm 1.47$ & $0.80 \pm 0.05$ \\
\hline & & & $27.13-49.13$ & $3.88-13.60$ & $5.00-13.85$ & $3.25-11.75$ & $0.40-1.17$ \\
\hline & \multirow{2}{*}{ wo } & \multirow{2}{*}{ F. Arno } & $64.13 \pm 3.71$ & $11.61 \pm 0.67$ & $11.83 \pm 1.48$ & $8.25 \pm 1.43$ & $0.73 \pm 0.04$ \\
\hline & & & $43.83-86.67$ & $6.38-17.44$ & $7.25-17.50$ & $3.75-13.25$ & $0.35-0.90$ \\
\hline & \multirow{2}{*}{ WI } & \multirow{2}{*}{ T. Oia } & $40.03 \pm 1.73$ & $10.17 \pm 0.59$ & $11.63 \pm 1.64$ & & \\
\hline & & & $29.33-49.21$ & $5.76-15.17$ & $4.50-15.50$ & $5.00-13.75$ & $0.49-1.01$ \\
\hline & \multirow{2}{*}{ WI } & \multirow{2}{*}{\begin{tabular}{|l} 
F. so \\
Prugnano
\end{tabular}} & $32.94 \pm 1.89$ & $9.54 \pm 0.56$ & $11.93 \pm 1.28$ & $8.05 \pm 1.42$ & \pm 0.06 \\
\hline & & & & 4.80 & & & \\
\hline \multirow{8}{*}{ 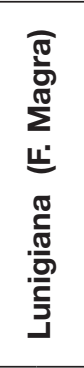 } & \multirow{2}{*}{ wo } & \multirow{2}{*}{ T. Aulella } & & 10.92 & 1.16 & & \\
\hline & & & 25.78 & 8.40 & 5.50 & 7.5 & 0.51 \\
\hline & \multirow{2}{*}{ wo } & \multirow{2}{*}{$\begin{array}{l}\text { F. so della } \\
\text { Pezzola }\end{array}$} & 39. & 9.80 & 10. & & \\
\hline & & & 23. & 8.58 & & & \\
\hline & \multirow{2}{*}{ WI } & \multirow{2}{*}{$\begin{array}{l}\text { F. so delle } \\
\text { Selve }\end{array}$} & $21.55 \pm 1.80$ & $10.80=$ & 11.60 & 10.25 & 0.3 \\
\hline & & & 31. & 7.75 & 25 & & \\
\hline & \multirow{2}{*}{ WI } & \multirow{2}{*}{$\begin{array}{l}\text { Canale di } \\
\text { Collegnago }\end{array}$} & & 11.1 & & & \\
\hline & & & 27.5 & 6.18 & 4.50 & & .86 \\
\hline \multirow{6}{*}{ 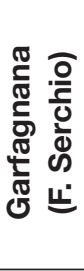 } & \multirow{2}{*}{ wo } & \multirow{2}{*}{$\begin{array}{l}\text { F. so } \\
\text { Gambrano }\end{array}$} & 30. & 10.4 & .30 & & \\
\hline & & & 16. & 7.19 & & & 1.72 \\
\hline & \multirow{2}{*}{ wo } & \multirow{2}{*}{\begin{tabular}{|l|} 
F. so i \\
Carpinelli \\
\end{tabular}} & $22.68 \pm 1.66$ & 10.36 & 1.16 & .70 & 0.05 \\
\hline & & & $29.52-39$. & 7.60 & 8.00 & 5.2 & 0.67 \\
\hline & \multirow{2}{*}{ WI } & \multirow{2}{*}{\begin{tabular}{|l} 
T. Acqua \\
Bianca
\end{tabular}} & 34.03 & 11.46 & 12. & & \\
\hline & & & $28.5 \xi$ & 6.45 & 3.00 & 25 & 0.50 \\
\hline \multirow{8}{*}{ 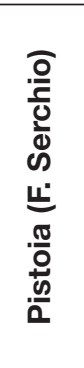 } & & & 36.04 & 11.04 & 11.1 & .41 & 0.04 \\
\hline & n & & 18.7 & 5.40 & .50 & .75 & 0.81 \\
\hline & Wo & & $39.68 \pm 2.20$ & $10.65 \pm 0.44$ & $11.39 \pm 1.16$ & .85 & 0.03 \\
\hline & (1) & 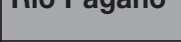 & $26.92-50.21$ & $5.40-17.41$ & $5.75-17.75$ & $4.50-14.00$ & $0.49-1.02$ \\
\hline & WI & & $30.44 \pm 1.64$ & $10.36 \pm 0.55$ & $11.19 \pm 1.19$ & 1.47 & $0.69 \pm 0.05$ \\
\hline & VVI & & 32.29 - & 6.57 & 15.00 & .75 & 0.60 \\
\hline & WI & & $28.04 \pm 1.99$ & $10.44 \pm 0.62$ & $11.61 \pm 1.61$ & $9.31 \pm 1.52$ & $0.45 \pm 0.05$ \\
\hline & & & $21.00-42.42$ & $5.76-14.97$ & $7.00-15.50$ & $3.50-13.75$ & $0.35-1.06$ \\
\hline & & & $44.85 \pm 2.40$ & $13.36 \pm 0.61$ & $14.65 \pm 1.55$ & $11.06 \pm 1.93$ & $0.58 \pm 0.08$ \\
\hline & & & $30.77-59.50$ & $8.00-17.65$ & $9.60-18.75$ & $3.75-16.75$ & $0.04-1.08$ \\
\hline$\frac{\omega}{\omega}$ & & T. & $24.96 \pm 2.04$ & $11.32 \pm 0.57$ & $13.63 \pm 1.46$ & $8.94 \pm 1.28$ & $0.56 \pm 0.07$ \\
\hline & & & $15.28-32.17$ & $6.65-15.48$ & $7.50-17.00$ & $4.75-13.50$ & $0.16-0.88$ \\
\hline 을 & WI & & $37.33 \pm 2.02$ & $12.00 \pm 0.57$ & $13.31 \pm 1.45$ & $10.10 \pm 1.16$ & $0.69 \pm 0.05$ \\
\hline & & & $25.13-49.83$ & $7.40-15.86$ & $8.25-16.50$ & $6.00-14.00$ & $0.34-0.91$ \\
\hline & & & $33.98 \pm 2.24$ & $12.01 \pm 0.61$ & $13.76 \pm 1.54$ & $9.61 \pm 1.48$ & $0.32 \pm 0.05$ \\
\hline & WI & & $24.21-45.98$ & $7.03-15.88$ & $8.50-16.50$ & $4.25-13.50$ & $0.01-0.59$ \\
\hline
\end{tabular}




\section{Table II}

Yearly mean values ( \pm standard deviation) and ranges of chemical parameters measured on every site. WI and WO denote the watercourses with and without A. pallipes complex.

Tableau II

Valeurs moyennes annuelles ( \pm écart-type) et gammes de valeurs des paramètres chimiques mesurés sur chaque site. WI et WO désignent respectivement les cours d'eau avec et sans le complexe A. pallipes.

\begin{tabular}{|c|c|c|c|c|c|c|c|c|c|}
\hline & & & $\mathrm{pH}$ & Cond $\left(\mu \mathrm{Scm}^{-1}\right)$ & $\mathrm{O}_{2}$ (mgl-1) & $\mathrm{NO}_{3}\left(\mathrm{mgl}^{-1}\right)$ & $\mathrm{NO}_{2}\left(\mathrm{mgl}^{-1}\right)$ & $\mathrm{Ca}\left(\mathrm{mgl}^{-1}\right)$ & $\mathrm{Si}\left(\mathrm{mgl}^{-1}\right)$ \\
\hline \multirow{8}{*}{ 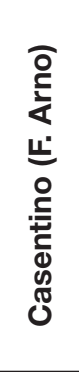 } & \multirow{2}{*}{ Wo } & F. so & $8.16 \pm 0.05$ & $248.54 \pm 4.20$ & $9.65 \pm 0.24$ & $6.69 \pm 0.26$ & $0.06 \pm 0.01$ & $45.75 \pm 0.88$ & $11.13 \pm 0.53$ \\
\hline & & Camaldoli & $7.97-8.33$ & $223.25-273.67$ & $8.80-11.83$ & $5.00-8.00$ & $0.04-0.08$ & $39.75-51.50$ & $6.83-13.87$ \\
\hline & \multirow{2}{*}{ Wo } & \multirow{2}{*}{ F. Arno } & $8.31 \pm 0.04$ & $331.29 \pm 4.45$ & $9.97 \pm 0.25$ & $2.56 \pm 0.28$ & $0.05 \pm 0.01$ & $59.04 \pm 1.01$ & $13.83 \pm 0.67$ \\
\hline & & & $8.19-8.38$ & $304.25-367.92$ & $8.98-11.61$ & $0.00-3.5$ & $0.04-0.08$ & $52.00-68.58$ & $7.50-18.67$ \\
\hline & \multirow{2}{*}{ WI } & \multirow{2}{*}{ T. Oia } & $8.09 \pm 0.04$ & $243.85 \pm 5.97$ & $9.89 \pm 0.26$ & $4.25 \pm 0.49$ & $0.05 \pm 0.01$ & $45.23 \pm 1.24$ & $12.24 \pm 0.63$ \\
\hline & & & $7.89-8.28$ & $205.50-274.83$ & $8.77-12.21$ & $0.00-8.00$ & $0.04-0.06$ & $37.83-53.50$ & $7.50-16.27$ \\
\hline & \multirow{2}{*}{ WI } & F. so & $7.97 \pm 0.06$ & $452.38 \pm 6.55$ & $8.50 \pm 0.30$ & $2.56 \pm 0.27$ & $0.06 \pm 0.01$ & $85.67 \pm 1.55$ & $11.44 \pm 0.64$ \\
\hline & & Prugnano & $7.64-8.36$ & $420.00-499.50$ & $6.57-11.40$ & $1.50-4.00$ & $0.04-0.08$ & $75.00-94.50$ & $7.38-16.20$ \\
\hline \multirow{8}{*}{ 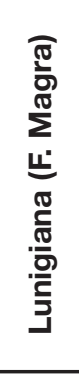 } & \multirow{2}{*}{ Wo } & \multirow{2}{*}{ T. Aulella } & $8.37 \pm 0.03$ & $340.08 \pm 5.98$ & $9.49 \pm 0.17$ & $4.35 \pm 0.30$ & $0.08 \pm 0.01$ & $67.00 \pm 0.85$ & $13.22 \pm 0.72$ \\
\hline & & & $8.13-8.52$ & $321.75-375.08$ & $7.91-9.37$ & $2.50-5.00$ & $0.04-0.06$ & $62.75-73.00$ & $9.54-24.00$ \\
\hline & \multirow{2}{*}{ wo } & F. so della & $8.02 \pm 0.05$ & $169.58 \pm 4.73$ & $9.11 \pm 0.13$ & $4.06 \pm 0.46$ & $0.05 \pm 0.01$ & $32.44 \pm 0.90$ & $12.28 \pm 0.52$ \\
\hline & & Pezzola & $8.28-8.59$ & $302.50-362.75$ & $7.61-10.45$ & $2.17-5.00$ & $0.04-0.08$ & $27.00-42.25$ & $9.25-24.33$ \\
\hline & \multirow{2}{*}{ WI } & F. so delle & $8.26 \pm 0.03$ & $347.58 \pm 4.03$ & $8.74 \pm 0.13$ & $3.88 \pm 0.38$ & $0.05 \pm 0.01$ & $67.75 \pm 0.94$ & $17.19 \pm 0.75$ \\
\hline & & Selve & $8.26-8.60$ & $304.00-391.50$ & $8.48-10.07$ & $2.42-5.00$ & $0.03-0.18$ & $64.75-72.50$ & $8.08-20.80$ \\
\hline & \multirow{2}{*}{ WI } & & $8.40 \pm 0.02$ & $334.15 \pm 4.38$ & $9.24 \pm 0.18$ & $4.29 \pm 0.31$ & $0.06 \pm 0.01$ & $72.75 \pm 1.10$ & $14.51 \pm 0.86$ \\
\hline & & Collegnago & $7.70-8.21$ & $141.00-219.25$ & $7.91-9.93$ & $2.50-6.25$ & $0.04-0.06$ & $62.50-77.75$ & $7.50-17.60$ \\
\hline \multirow{6}{*}{ 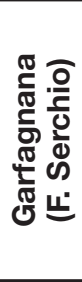 } & \multirow{2}{*}{ wo } & & $8.33 \pm 0.03$ & $394.50 \pm 2$ & $8.94 \pm 0.19$ & $3.56 \pm 0.31$ & $0.07 \pm 0.01$ & 83.83 & $13.32 \pm 0.37$ \\
\hline & & Gambrano & $8.19-8.49$ & $339.83-406.42$ & $7.91-10.18$ & $1.67-5.00$ & $0.03-0.07$ & $78.08-89.00$ & $8.67-17.60$ \\
\hline & \multirow{2}{*}{ wo } & F. so i & $8.30 \pm 0.03$ & $373.15 \pm 3.78$ & $9.15 \pm 0.17$ & $3.23 \pm 0.40$ & $0.05 \pm 0.01$ & $76.90 \pm 0.96$ & $12.57 \pm 0.48$ \\
\hline & & Carpinelli & $8.28-8.68$ & $218.50-345.50$ & $8.19-10.46$ & $0.75-5.00$ & $0.05-0.13$ & $72.25-80.00$ & $8.67-17.60$ \\
\hline & \multirow{2}{*}{ WI } & T. Acqua & $8.50 \pm 0.03$ & $260.38 \pm 7.87$ & $9.32 \pm 0.17$ & $3.94 \pm 0.37$ & $0.07 \pm 0.01$ & $51.92 \pm 1.28$ & $12.57 \pm 0.48$ \\
\hline & & & $8.15-8.59$ & $382.08-418.42$ & $7.66-10.66$ & $0.50-5.00$ & $0.05-0.15$ & $45.08-64.75$ & $11.67-17.60$ \\
\hline \multirow{8}{*}{ 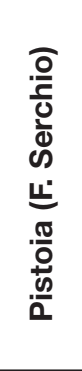 } & \multirow{2}{*}{ Wo } & F. so della & $7.83 \pm 0.06$ & $146.27 \pm 6.43$ & $9.38 \pm 0.21$ & $3.00 \pm 0.36$ & $0.05 \pm 0.01$ & $25.56 \pm 1.21$ & $11.41 \pm 0.47$ \\
\hline & & & $7.99-8.74$ & $316.25-449.50$ & $7.28-10.99$ & $3.00-7.50$ & $0.03-0.07$ & $17.58-31.50$ & $6.83-14.80$ \\
\hline & \multirow{2}{*}{ wo } & \multirow{2}{*}{ Rio Pagano } & $8.15 \pm 0.05$ & $256.92 \pm 4.16$ & $8.94 \pm 0.22$ & $5.06 \pm 0.49$ & $0.05 \pm 0.01$ & $47.29 \pm 0.93$ & $13.39 \pm 0.61$ \\
\hline & & & $7.57-8.08$ & $104.58-184.50$ & $7.70-11.42$ & $1.00-5.00$ & $0.04-0.06$ & $39.25-54.33$ & $6.83-14.80$ \\
\hline & \multirow{2}{*}{ WI } & Rio & $7.97 \pm 0.06$ & $208.02 \pm 7.65$ & $9.42 \pm 0.18$ & $4.81 \pm 0.35$ & $0.05 \pm 0.01$ & $38.46 \pm 1.52$ & $11.49 \pm 0.45$ \\
\hline & & D'Omicio & $7.73-8.56$ & $233.75-296.17$ & $7.30-10.52$ & $1.50-7.00$ & $0.03-0.08$ & $28.25-47.08$ & $8.96-19.20$ \\
\hline & \multirow{2}{*}{ WI } & F. so & $8.32 \pm 0.04$ & $384.94 \pm 9.73$ & $9.08 \pm 0.22$ & $5.00 \pm 0.34$ & $0.05 \pm 0.01$ & $70.71 \pm 1.63$ & $11.41 \pm 0.47$ \\
\hline & & & $7.79-8.16$ & $154.00-250.42$ & $8.12-11.06$ & $1.75-7.50$ & $0.05-0.06$ & $60.00-80.75$ & $7.17-14.80$ \\
\hline \multirow{8}{*}{ 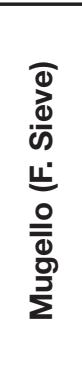 } & \multirow{2}{*}{ wo } & & $8.09 \pm 0.05$ & $535.65 \pm 3.16$ & $9.56 \pm 0.22$ & $6.38 \pm 0.29$ & $0.08 \pm 0.01$ & $76.02 \pm 3.27$ & $18.00 \pm 1.02$ \\
\hline & & 1. Carza & $7.72-8.44$ & $512.00-561.25$ & $7.86-11.57$ & $4.25-7.50$ & $0.06-0.15$ & $52.00-101.00$ & $11.25-27.50$ \\
\hline & & T. & $8.21 \pm 0.05$ & $560.56 \pm 13.29$ & $9.24 \pm 0.25$ & $6.63 \pm 0.44$ & $0.13 \pm 0.01$ & $80.60 \pm 2.21$ & $16.96 \pm 0.86$ \\
\hline & vo & & $7.87-8.66$ & $477.00-628.25$ & $7.46-11.45$ & $3.50-9.00$ & $0.08-0.21$ & $71.25-88.67$ & $12.93-23.90$ \\
\hline & WI & T Muccione & $8.28 \pm 0.05$ & $460.60 \pm 3.44$ & $9.12 \pm 0.21$ & $5.25 \pm 0.47$ & $0.08 \pm 0.01$ & $66.00 \pm 0.46$ & $14.58 \pm 0.73$ \\
\hline & $\mathbf{W I}$ & 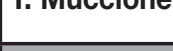 & $8.03-8.47$ & $440.25-490.08$ & $7.76-10.85$ & $2.50-8.00$ & $0.05-0.11$ & $64.42-67.83$ & $9.25-20.80$ \\
\hline & & F. so & $8.04 \pm 0.07$ & $458.94 \pm 18.05$ & $9.17 \pm 0.24$ & $4.31 \pm 0.39$ & $0.09 \pm 0.01$ & $73.27 \pm 1.12$ & $12.84 \pm 0.52$ \\
\hline & VI & Farfereta & $7.56-8.73$ & $405.75-513.83$ & $7.48-11.32$ & $2.25-6.50$ & $0.04-0.11$ & $67.50-80.25$ & $8.38-17.60$ \\
\hline
\end{tabular}




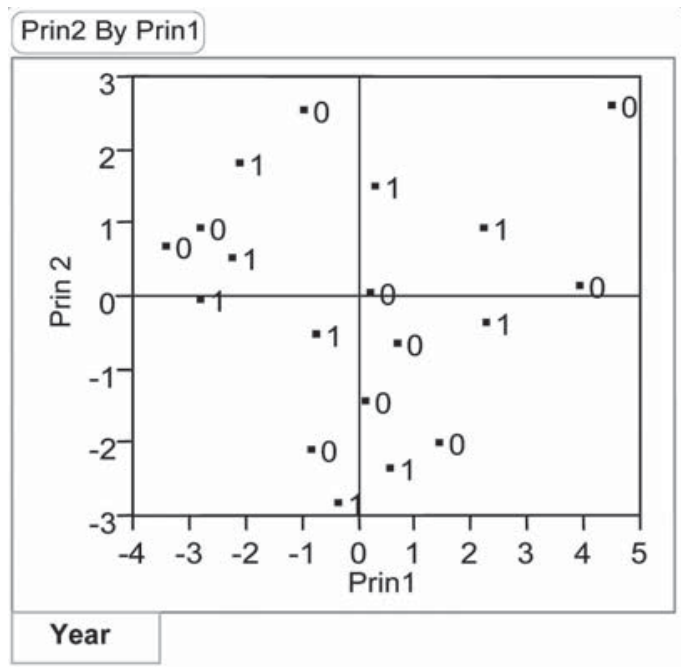

Figure 2

Scatterplot of the two principal components of yearly mean values of physicochemical variables. 1 and 0 denote the watercourses with and without $A$. pallipes complex.

\section{Figure 2}

Représentation graphique des deux composantes principales construites à partir des valeurs moyennes annuelles des variables physico-chimiques. 1 et 0 désignent respectivement les ruisseaux avec et sans le complexe $A$. pallipes.

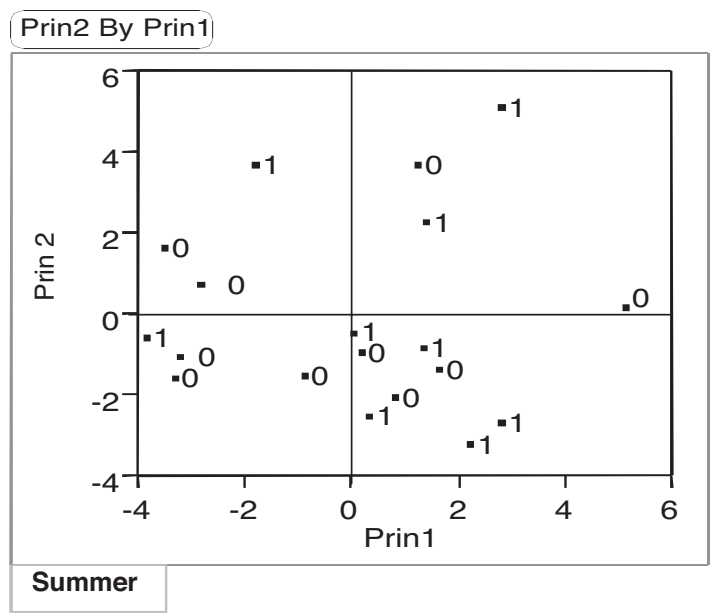

Figure 3

Scatterplot of the two principal components of summer mean values of biotic and abiotic variables. 1 and 0 denote the watercourses with and without $A$. pallipes complex.

\section{Figure 3}

Représentation graphique des deux composantes principales construites à partir des valeurs moyennes estivales des variables biotiques et abiotiques. 1 et 0 désignent respectivement les ruisseaux avec et sans le complexe $A$. pallipes. 


\section{Table III}

Mean values of $\mathrm{PC}_{1}$ and $\mathrm{PC}_{2}$ between streams with (WI) and without (WO) crayfish. Year, Spring, Summer, Autumn and Winter: PCA of mean values of physico-chemical variables; Summer (abiotic and biotic parameters): PCA of summer mean values of physico-chemical parameters integrated with biotic, IBE and IFF data.

Tableau III

Valeurs moyennes de $\mathrm{PC}_{1}$ et $\mathrm{PC}_{2}$ entre les ruisseaux avec (WI) et sans (WO) écrevisses. ACP des valeurs moyennes des paramètres physico-chimiques: "year" pour annuelle, "spring" pour printemps, "summer" pour été, "autumn" pour automne et "winter" pour hiver. L'ACP de l'été avec les paramètres abiotiques et biotiques est construite avec les valeurs moyennes des paramètres physicochimiques et avec les valeurs des indices biotiques IBE et IFF.

\begin{tabular}{|c|c|c|c|c|}
\hline & \multicolumn{2}{|c|}{$\mathrm{PC}_{1}$} & \multicolumn{2}{|c|}{$\mathrm{PC}_{2}$} \\
\hline \multirow{2}{*}{ Year } & $\mathbf{W I}=-0.317 \pm 0.75$ & W0 $=0.285 \pm 0.71$ & $\mathbf{W I}=-0.123 \pm 0.55$ & W0 $=0.110 \pm 0.52$ \\
\hline & \multicolumn{2}{|c|}{$P>0.1$} & \multicolumn{2}{|c|}{$P>0.1$} \\
\hline \multirow{2}{*}{ Spring } & $\mathbf{W I}=0.015 \pm 0.75$ & W0 $=-0.014 \pm 0.71$ & $\mathbf{W I}=-0.241 \pm 0.47$ & W0 $=0.217 \pm 0.45$ \\
\hline & \multicolumn{2}{|c|}{$P>0.1$} & \multicolumn{2}{|c|}{$P>0.1$} \\
\hline \multirow{2}{*}{ Summer } & $\mathbf{W I}=0.178 \pm 0.65$ & W0 $=-0.160 \pm 0.61$ & $\mathbf{W I}=-0.321 \pm 0.61$ & W0 $=0.289 \pm 0.58$ \\
\hline & \multicolumn{2}{|c|}{$P>0.1$} & \multicolumn{2}{|c|}{$P>0.1$} \\
\hline \multirow{2}{*}{ Autumn } & $\mathbf{W I}=0.330 \pm 0.78$ & W0 $=-0.297 \pm 0.74$ & $\mathbf{W I}=-0.096 \pm 0.52$ & W0 $=0.086 \pm 0.49$ \\
\hline & \multicolumn{2}{|c|}{$P>0.1$} & \multicolumn{2}{|c|}{$P>0.1$} \\
\hline \multirow{2}{*}{ Winter } & $\mathbf{W I}=0.278 \pm 0.71$ & W0 $=-0.250 \pm 0.68$ & $\mathbf{W I}=0.109 \pm 0.56$ & WO $=-0.098 \pm 0.53$ \\
\hline & \multicolumn{2}{|c|}{$P>0.1$} & \multicolumn{2}{|c|}{$P>0.1$} \\
\hline \multirow{2}{*}{$\begin{array}{l}\text { Summer } \\
\text { (abiotic } \\
\text { and biotic } \\
\text { parameters) }\end{array}$} & $\mathbf{W I}=0.544 \pm 0.85$ & W0 $=-0.499 \pm 0.80$ & $\mathbf{W I}=0.160 \pm 0.80$ & W0 $=-0.144 \pm 0.76$ \\
\hline & \multicolumn{2}{|c|}{$P>0.1$} & \multicolumn{2}{|c|}{$P>0.1$} \\
\hline
\end{tabular}

\section{DISCUSSION}

\section{Environmental differences between watercourses}

Previous studies on A. pallipes (LAURENT, 1985; FOSTER and TURNER, 1993; TROSCHEL, 1997; BROQUET et al., 2002; TROUILHE et al., 2003) did not succeed in establishing a clear relationship between the presence of crayfish and different values of physico-chemical parameters. These results could be an effect of the methods of analysis used. An alternative explanation is that $A$. pallipes may not be a good bioindicator of good environmental quality (TROSCHEL, 1997; GRANDJEAN et al., 2000, 2001; BROQUET et al., 2002; DEMERS and REYNOLDS, 2002; TROUILHE et al., 2003), as commonly hypothesized. The species is mostly found in clean waters; it can also survive in waters where intermittent pollution occurs, indicating that its presence does not only depend on water chemistry (HOLDICH and REEVE, 1991; TROSCHEL, 1997; DEMERS and REYNOLDS, 2003).

Using Principal Components Analysis, we were not able to separate between watercourses with and without $A$. pallipes complex. Indeed, the streams where crayfish were absent displayed physico-chemical values similar to those required by the species and had high values of IBE (I-II). They were habitats of good quality and showed availability of shelters and canopy cover favourable to the survival of this crayfish. 
Several studies (ARRIGNON and ROCHÉ, 1983; TROSCHEL, 1993, 1997, 2002; FOSTER, 1995; NEVEU, 2000 a,b; MARTİNEZ et al., 2003) showed that the suitable environment for $A$. pallipes is constituted by lotic waters with moderate currents, running through mountainous or hilly areas and forests where no pollution occurs. Generally, all small watercourses rich in stones, roots, and decaying wood, with a maximum width of $5 \mathrm{~m}$ and a depth of about $1 \mathrm{~m}$ are inhabited by this species (GRANDJEAN et al., 1996; BERNARDO et al., 1997; TROSCHEL, 1997; GARCìA-ARBERAS and RALLO, 2000). In fact, crayfish find abundant hiding places, according to their size, among tree roots, dead wood, and stones, whereas the availability of places to dig themselves into a hole is generally very rare (TROSCHEL, 1997).

Our study sites showed all these physical and biotic features and the water was always well oxygenated $\left(6.57-12.21 \mathrm{mgl}^{-1}\right)$ with values slightly higher than studied French watercourses (GRANDJEAN et al., 2001) (7-10 mgl-1). Dissolved oxygen concentration plays a leading role in freshwater habitat and it has been found to be critical in the successful culture of several crayfish species (ACKEFORS, 1996). Conductivity values in Tuscan streams were similar to those recorded by GRANDJEAN et al. (2001) (200$\left.600 \mu \mathrm{Scm}^{-1}\right)$ in France and by RALLO and GARCİA-ARBERAS (2002) (144-607 $\left.\mu \mathrm{Scm}^{-1}\right)$ in some northern rivers of the Iberian Peninsula. However, their range differed slightly from the ones found in other countries (France: 310-385 $\mu \mathrm{Scm}^{-1}$, ARRIGNON et al., 1993; Germany: 225-345 $\mu \mathrm{Scm}^{-1}$, TROSCHEL, 1997; Spain: 457-1638 $\mu \mathrm{Scm}^{-1}$, ALONSO, 2001; Britain: 93-643 $\mu \mathrm{Scm}^{-1}$, SMITH et al., 1996; Wales: 60-390 $\mu \mathrm{Scm}^{-1}$, LILLEY et al., 1979). The conductivity range was rather high, but this variable does not seem to be a limiting factor for $A$. pallipes. In fact, this species has been found in ponds with water well above $400 \mu \mathrm{Scm}^{-1}$ (BOHL, 1997) and living at more than $1700 \mu \mathrm{Scm}^{-1}$ in diapiric holes (RALLO and GARCİA-ARBERAS, 2000). Several differences appeared also in the maximum water temperature (Italy: $18.75^{\circ} \mathrm{C}$; France: $<18^{\circ} \mathrm{C}$; Germany: $16.8^{\circ} \mathrm{C}$; Spain: $24^{\circ} \mathrm{C}$ ) and in mean water speed that was higher than German watercourses $(0.1-0.8 \mathrm{~m} / \mathrm{sec}$ ) (TROSCHEL, 1997). Probably the altitude and the geological characteristics of the other regions compared with Tuscany could explain some differences. Conductivity depends on geological formation at the river catchments.

Crayfish are said to live in a range of 6.5-9.0 pH (CHAISEMARTIN, 1967; HOLDICH and JAY, 1977). The values of $\mathrm{pH}$ we measured in each Tuscan watercourse were comprised in this range. They were slightly higher than the values recorded in German sites (6.8-8.3) (TROSCHEL, 1997) and in the Pays de Loire region (7.45) (BROQUET et al., 2002), but similar to the $\mathrm{pH}$ range found in the Deux-Sèvre department (western France, 7.6-8.7) (TROUILHE et al., 2003), in England (7-9) (LOWERY, 1988), in central Wales (7.278.64) (FOSTER, 1995), in Basque Country (Spain) (7.95-8.45) (GARCl̀A-ARBERAS and RALLO, 2000), and in some rivers of the Iberian Peninsula (7.6-8.1) (RALLO and GARCìAARBERAS, 2002).

$\mathrm{Ca}^{2+}$ and $\mathrm{Mg}^{2+}$ are other important factors associated with crayfish presence since they are essential components of their integument and are required for successful growth and moulting (JUSSILA et al., 1995). CHAISEMARTIN (1967) and HOLDICH and JAY (1977) observed that levels higher than $5 \mathrm{mgl}^{-1}$ are required for crayfish survival. The measures of calcium concentration were rather high (17.58-101.00 $\left.\mathrm{mgl}^{-1}\right)$ in both WI and WO watercourses.

\section{Possible causes of $A$. pallipes complex local extinctions}

In Europe the decline of indigenous crayfish was mainly related to the introduction of alien species such as Procambarus clarkii that outcompetes other crayfish species (HOLDICH and REEVE, 1991; GIL-SÀNCHEZ and ALBA-TERCEDOR, 2002) and is a vector of aphanomycosis (DIÉGUEZ-URIBEONDO and SÖDERHÄL, 1993; DIÉGUEZURIBEONDO et al., 1995), lethal to the European species of Astacidae (ALDERMAN and 
POLGLASE, 1988; GHERARDI and HOLDICH, 1999). Fortunately in recent years, epidemics of plague were not found in Italy (F. QUAGLIO, pers. com.) and in Tuscany P. clarkii and $A$. pallipes never cohabited in the same reaches. Recently, sympatric populations of the two species were observed in a watercourse (L. AQUILONI, pers. com.), but $P$. clarkii is distributed only along the lower stretches.

In Tuscany, the effects of the heat wave of summer 2003, causing near desiccation of some watercourses, should be considered as a probable explanation of a reduction in abundance of the population below the level of detection or perhaps the cause of some local extinctions, even if this cannot explain the complete extinction of many populations. The headwaters that have potentially good physical habitat for crayfish can be adversely affected by reduced base flow due to abstraction of ground water for public water supply. This means that these streams have low flows and are at risk of pollution from small domestic discharges. In some years the whole channel may dry up completely and the availability of in-channel habitat is rather low. When the shelters are exposed to air, the crayfish have to move out into water, especially in hot conditions. Since most refuges are in the banks, moving into the mid channel will leave crayfish very vulnerable to desiccation and/or predation.

GIL-SÀNCHEZ and ALBA-TERCEDOR (2002) refer to water abstraction for human use, drought and poor water quality as contributing factors to the loss of $A$. pallipes populations although they stress that introduced crayfish species are probably the dominant factor.

HOGGER (1988) showed that not many of the potential crayfish predators were known to affect crayfish abundance with the exception of fish (ENGLUND, 1999). However in the watercourses of our study there are no or very few predators.

The localised and sporadic pollution is also an important factor influencing crayfish survival. In fact, any sporadic event of low dissolved oxygen concentration is relevant to crayfish, especially with a spike of high BOD or ammonia. In this case it would be useful to have some historical data, especially for periods before and after crayfish disappeared to identify the main cause of crayfish extinction. The presence of sporadic pollution emerged from interviews only for Fosso Gambrano (Lucca). Some old people remembered that organic discharges from pig-breeding were released into the watercourse with high densities of crayfish. Pig manure has a high ammonia content and is potentially toxic to crayfish, in addition to the effect of decomposition of organic matter on dissolved oxygen. FOSTER $(1991,1995)$ brings up the issue of impact of localised pollution and SLATER (2001) described one event with sheep-dip in 1997 that estirpated all the crayfish on a stretch of the Afon Sgithwen. By 1998 and 1999 the macroinvertebrate fauna was completely recovered, but no crayfish were found. This could be the same case for Fosso Gambrano watercourse, which scores I for IBE value but shows the absence of this species.

Moreover in Tuscany freshwater crayfish has no economic value but have been exploited for centuries being recognized as a delicacy. Due to this phenomenon, legislative measures were adopted and the harvesting of Austropotamobius pallipes complex is prohibited by the L.R. 7/2005.

We hypothesize that both pollution incidents and over-exploitation through illegal fishing for local use might have affected the abundance of crayfish, and have caused their extinction. In fact at present Tuscan populations of $A$. pallipes complex are commonly found in small creeks, frequently isolated by permanent barriers from the main river system, and they occupy small areas (B. RENAI, unpublished data). The same was observed by some authors in several Spanish regions (GAUDIOSO et al., 1987; ALDABE et al., 1991; CARRAL et al., 1993; BOLEA, 1995) and in the Basque Country (GARCÌA-ARBERAS and RALLO, 2000). 


\section{CONCLUSIONS}

Our results show that the disappearance of crayfish populations that we recorded in several watercourses in Tuscany is not due to habitat degradation or alteration, although, historically, pollution incidents and/or drought events may have had an impact. The macroinvertebrate fauna shows that pollution is not a contraint at present. These streams, displaying the ecological requirements for this species, are ideal for programs of reintroduction.

Among the other factors that may cause crayfish extinction, alien crayfish were not found in the study sites. However, a high level of attention towards the problem of introduction of invasive species is necessary. Risks may come from the narrowing of the distribution area of the species and from the isolation among populations and their generally low densities (B. RENAl, unpublished data).

Finally, studies to implement information on the biology and growth of $A$. pallipes complex should be intensified to improve the measures of protection. Moreover surveys should be carried out on the development of populations following reintroductions, as this may help to improve method and the success of future introductions or the conservation of the native crayfish.

\section{ACKNOWLEDGEMENTS}

This work forms part of the Galileo project France/Italy, 2004-2005: "Environmental quality of freshwaters for the conservation and management of the threatened crayfish (genus Austropotamobius)". It was made possible by grants from UN.D.A. FELIX for the environmental studies and from the Italian Ministry of Agriculture (MIPAF, "Sesto Piano Triennale per la Pesca e l'Acquacoltura").

\section{REFERENCES}

ACKEFORS H., 1996. The development of crayfish culture in Sweden during the last decade. Freshwater Crayfish, 11, 627-654.

ALDABE J., ESQUISABEL J.I., FERNÁNDEZ M.J., IRURZUN J.C., MENDÍA F., RUEDA A., YOLDI B., 1991. Estudio de distribucion y abundancia de las especies de cangrejos presentes en los rios navarros. Internal report of the Servicio de Medio Ambiente del Gobierno de Navarra, Negociado de Gestión Cinegética y Piscícola. (In Spanish).

ALDERMAN D.J., POLGLASE J.L., 1988. Pathogens, parasites and commensals. In: HOLDICH D.M. and LOWERY R.S. (eds.), Freshwater Crayfish: Biology, Management and Exploitation, 167-212, Croom Helm, London.

ALONSO F., 2001. Efficiency of electrofishing as a sampling method for freshwater crayfish populations in small creeks. Limnetica, 20(1), 59-72.

ANPA, 2003. IFF Indice di funzionalità fluviale - Manuale ANPA (Agenzia Nazionale per la Protezione dell'Ambiente)- 2a Edizione, Giugno 2003.

ARRIGNON J.C.V., ROCHÉ B., 1983. Population of the crayfish Austropotamobius pallipes pallipes (Lereboullet) in a brook of Corsica (France). Freshwater Crayfish, 5, 229238.

ARRIGNON J.C.V., LAMY G., ROCHÉ B., 1993. Situation d'une population corse d'Austropotamobius pallipes pallipes Lereb. Publ. Assoc. Dev. Aquac., 35, 89-98.

BERNARDO J.M., ILHEU M., COSTA A.M., 1997. Distribution, population structure and conservation of Austropotamobius pallipes in Portugal. Bull. Fr. Pêche Piscic., 347, 617-624. 
BOHL E., 1987. Comparative studies on crayfish brooks in Bavaria (Astacus astacus L., Austropotamobius torrentium Schr.). Freshwater Crayfish, 7, 287-294.

BOHL E., 1997. An isolated population of the white-clawed crayfish (Austropotamobius pallipes) in the principality of Liechtenstein. Bull. Fr. Pêche Piscic., 347, 701-712.

BOLEA L., 1995. El cangrejo de río común en Aragón. Diputación General de Aragón. Zaragoza. 64 p. (In Spanish).

BROQUET T., THIBAULT M., NEVEU A., 2002. Distribution and habitat requirements of the white-clawed crayfish, Austropotamobius pallipes, in a stream from the Pays de Loire region, France: an experimental and descriptive study. Bull. Fr. Pêche Piscic., 367, 717-728.

CARRAL J.M., CELADA J., GONZALEZ J., SAEZ-ROYUELA M., GAUDIOSO V.R., FERNÁNDEZ R., LÓPEZ-BAISSON C., 1993. Wild freshwater crayfish populations in Spain. Freshwater Crayfish, 9, 158-162.

CHAISEMARTIN C., 1967. Contribution à l'étude de l'économie calcique chez les Astacidae. Thèse de Doctorat Sci. Nat. Université de Poitiers.

DALE V.H., BEYELER S.C., 2001. Challenges in the development and use of ecological indicators. Ecol. Indicator, 1, 3-10.

DEMERS A., REYNOLDS J.D., 2002. A survey of the white-clawed crayfish, Austropotamobius pallipes (Lereboullet), and of water quality in two catchments of Eastern Ireland. Bull. Fr. Pêche Piscic., 367, 729-740.

DEMERS A., REYNOLDS J.D., 2003. The distribution of the white-clawed crayfish, Austropotamobius pallipes in eight catchments in Ireland in relation to water quality. In: HOLDICH D.M. and SIBLEY P.J. (eds.), Management and Conservation of Crayfish, 94-103, Proceedings of a conference held on $7^{\text {th }}$ November, 2002, Environment Agency, Bristol.

DEMERS A., REYNOLDS J.D., CIONI A., 2003. Habitat preference of different size classes of Austropotamobius pallipes in an Irish river. Bull. Fr. Pêche Piscic., 370-371, 127137.

DIÉGUEZ-URIBEONDO J., SÖDERHÄL K., 1993. Procambarus clarkii Girard as a vector for the crayfish plague fungus Aphanomyces astaci Schikora. Aquac. Fish. Manag., 24, 761-765.

DIÉGUEZ-URIBEONDO J., HUANG T., CERENIUS T., SÖDERHÄL K., 1995. Physiological adaptation of an Aphanomyces astaci strain isolated from the freshwater crayfish Procambarus clarkii. Mycol. Res., 99, 574-578.

DIÉGUEZ-URIBEONDO J., RUEDA A., CASTIEN E., BASCONES J.C., 1997. A plan of restoration in Navarra for the native freshwater crayfish species of Spain, Austropotamobius pallipes. Bull. Fr. Pêche Piscic., 347, 625-638.

DISTEFANO R.J., DECOSKE J.J., VANGILDER T.M., BARNES L.S., 2003. Macrohabitat partitioning among three crayfish species in two Missouri streams, U.S.A. Crustaceana, 76(3), 342-362.

ENGLUND G., 1999. Effects of fish on the local abundance of crayfish in stream pools. Oikos, 87, 48-56.

FLINT R.W., GOLDMAN C.R., 1977. Crayfish growth in Lake Tahoe: Effects of habitat variation. J. Fish Res. Board Can., 34, 155-159.

FOSTER J., 1991. Conservation and ecology of the crayfish Austropotamobius pallipes (Lereboullet) in Wales and the Marches in relation to habitat, water quality and 
the threat of crayfish plague. Report for the Worldwide Fund for Nature (Contract 89/87), University of Wales, UK.

FOSTER J., 1995. Factors influencing the distribution and abundance of the crayfish Austropotamobius pallipes (Lereboullet) in Wales and the Marches, UK. Freshwater Crayfish, 8, 78-98.

FOSTER J., TURNER C., 1993. Toxicity of field simulated farm waste episodes to the crayfish Austropotamobius pallipes (Lereboullet): elevated ammonia and reduced dissolved oxygen concentrations. Freshwater Crayfish, 9, 249-258.

FRATINI S., ZACCARA S., BARBARESI S., GRANDJEAN F., SOUTY-GROSSET C., CROSA G., GHERARDI F., 2004. Phylogeography of the threatened crayfish (genus Austropotamobius) in Italy: implications for its conservation. Heredity, 94, 108118.

FÜREDER L., OBERKOFLER B., HANEL R., MACHINO Y., 2002. Freshwater crayfish in South Tyrol (Italy): distribution and protection measures of endangered Austropotamobius pallipes. Bull. Fr. Pêche Piscic., 367, 651-662.

GARCìA-ARBERAS L., RALLO A., 2000. Survival of natural populations of Austropotamobius pallipes in rivers in Bizcaia, Basque Country (North Iberian Peninsula). Bull. Fr. Pêche Piscic., 356(1), 17-30.

GAUDIOSO V.R., CELADA J.D., CARRAL J., RODRÍGUEZ P.L., 1987. El cangrejo de rio en León. Universidad de León, León (In Spanish).

GHERARDI F., HOLDICH D.M., 1999. Crayfish in Europe as alien species. How to make the best of a bad situation? Crustacean Issues, 11. A.A. Balkema, Rotterdam, Brookfield, pp. 1-299.

GHETTI P.F., 1997. Indice Biotico Esteso (I.B.E.). I macroinvertebrati nel controllo della qualità degli ambienti di acque correnti. Manuale di applicazione. Provincia Autonoma di Trento, Trento, pp. 1-222.

GIL-SÀNCHEZ J.M., ALBA-TERCEDOR J., 2002. Ecology of the native and introduced crayfishes Austropotamobius pallipes and Procambarus clarkii in southern Spain and implications for conservation of the native species. Biol. Conserv., 105, 7580.

GODDARD J.S., 1988. Food and feeding. In: HOLDICH D.M. and LOWERY R.S. (eds.), Freshwater Crayfish: Biology, Management and Exploitation, 145-166, Croom Helm, London.

GODDARD J.S., HOGGER J.B., 1986. The current status and distribution of freshwater crayfish in Britain. Field Stud., 6, 383-96.

GRANDJEAN F., BRAMARD M., SOUTY-GROSSET C., 1996. Distribution and proposal for the conservation of the indigenous freshwater crayfish species, Austropotamobius pallipes pallipes, in a French department. Freshwater Crayfish, 11, 655-664.

GRANDJEAN F., CORNUAULT B., ARCHAMBAULT S., BRAMARD M., OTREBSKY G., 2000. Life history and population biology of the white-clawed crayfish, Austropotamobius pallipes, in a brook from the Poitou-Charentes region (France). Bull. Fr. Pêche Piscic., 356(1), 55-70.

GRANDJEAN F., JOUTEUX R., ROPIQUET A., BACHELIER E., BRAMARD M., OTREBSKY G., 2001. Biological water quality assessment in running waters sites harbouring the endangered crayfish, Austropotamobius pallipes based on macroinvertebrates and its potential in habitat selection for restocking. Freshwater Crayfish, 13, 338348. 
HOGGER J.B., 1984. A study of aspects of the biology and distribution of freshwater crayfish in the Thames catchment. Unpublished Ph.D. Thesis, CNAA, UK.

HOGGER J.B., 1988. Ecology, population biology and behaviour. In: HOLDICH D.M. and LOWERY R.S. (eds.), Freshwater Crayfish: Biology, Management and Exploitation, 114-144, Croom Helm, London \& Sydney.

HOLDICH D.M., JAY D., 1977. The pH tolerance of the crayfish Austropotamobius pallipes (Lereboullet). Freshwater Crayfish, 3, 363-370.

HOLDICH D.M., REEVE I.D., 1987. Status of native crayfish with particular reference to crayfish plague, alien introductions and pollution. Report to the Nature Conservancy Council, Contract No. HF3-030208(11), UK.

HOLDICH D.M., REEVE I.D., 1991. Distribution of freshwater crayfish in the British Isles, with particular reference to crayfish plague, alien introductions and water quality. Aquat. Conserv.: Mar. Freshwat. Ecosyst., 1, 139-158.

HOLDICH D.M., ROGERS W.D., 1997. The white-clawed crayfish, Austropotamobius pallipes, in Great Britain and Ireland with particular reference to its conservation in Great Britain. Bull. Fr. Pêche Piscic., 347, 597-616.

HOLDICH D.M., JAY D., GODDARD J.S., 1978. Crayfish in the British Isles. Aquaculture, $15,91-97$.

HUXLEY T.H., 1879. The crayfish. An introduction to the study of zoology. Keegan Paul, London.

IUCN (INTERNATIONAL UNION FOR CONSERVATION), 1996. In: BAILLIE J. and GROOMBRIDGE B. (eds.), 1996 IUCN Red list of Threatened Animals. IUCN, Gland, Switzerland.

JAY D., HOLDICH D.M., 1981. The distribution of the crayfish, Austropotamobius pallipes, in British waters. Freshwater Biol., 11, 121-129.

JUSSILA J., HENTTONEN P., HUNER J.V., 1995. Calcium, magnesium and manganese content of the noble crayfish Astacus astacus (L.) branchial carapace and its relationships to water and sediment mineral contents of two ponds and one lake in central Finland. Freshwater Crayfish, 10, 230-238.

KAUSHIK N.K., BIRD G.A., 1987. Processing of maple, leaf, grass and fern packs and their colonisation by invertebrates in a stream. J. Freshwater Ecol., 4, 177-189.

KIRJAVAINEN J., WESTMAN K., 1999. Natural history and development of the introduced signal crayfish, Pacifastacus leniusculus, in a small, isolated Finnish lake, from 1968 to 1993. Aquat. Liv. Res., 12, 387-401.

LAURENT P.J., 1985. Une station d'écrevisses à pieds blancs: Austropotamobius pallipes Lere. (Decapoda-Astacidae) en zone périurbaine. Bull.Soc. Linn. Lyon, 3, 77-88.

LAURENT P.J., 1988. Austropotamobius pallipes and A. torrentium, with observations on their interactions with other species in Europe. In: HOLDICH D.M. and LOWERY R.S. (eds.), Freshwater Crayfish: Biology, Management and Exploitation, 341-364, University Press, Cambridge.

LIGHT T., ERMAN D.C., MYRICK C., CLARKE J., 1995. Decline of the Shasta crayfish (Pacifastacus fortis Faxon) of Northeastern California. Conserv. Biol., 9, 15671577.

LILLEY A.J., BROOKER M.P., EDWARDS R.W., 1979. The distribution of the crayfish, Austropotamobius pallipes (Lereboullet), in the upper Wye catchment, Wales. Nat. Wales 16(3), 195-200. 
LIU H., AVAULT J.W., MEDLEY P., 1995. Toxicity of ammonia and nitrite to juvenile redclaw crayfish, Cherax quadricarinatus (von Martens). Freshwater Crayfish, 10, 249-255.

LODGE D.M., HILL A.M., 1994. Factors governing species composition, population size and productivity of cool-water crayfishes. Nord. J. Freshwater Res., 69, 111-136.

LOWERY R.S., 1988. Growth, Moulting and Reproduction. In: HOLDICH D.M. and LOWERY R.S. (eds.), Freshwater Crayfish: Biology, Management and Exploitation, 83-144, Croom Helm, London.

MARCO A., QUILCHANO C., BLAUSTEIN A.R., 1999. Sensitivity to nitrate and nitrite in pond-breeding amphibians from the Pacific northwest. USA. Environ. Toxicol. Chem., 18, 2836-2839.

MARTİNEZ R., RICO E., ALONSO F., 2003. Characterisation of Austropotamobius italicus (Faxon, 1914) populations in a central Spain area. Bull. Fr. Pêche Piscic., 370-371, 43-56.

MASON J.C., MACDONALD S.M., 1982. The input of terrestrial invertebrates from tree canopies to a stream. Freshwater Biology, 12, 305-331.

MATTHEWS M.A., REYNOLDS J.D., 1995. A population study of the white-clawed crayfish Austropotamobius pallipes (Lereboullet) in an Irish reservoir. Biol. Environ.: Proc. $R$. Ir. Acad., 95B, 99-109.

MOMOT W.T., 1984. Crayfish production: a reflection of community energetics. J. Crustacean Biology, 4, 35-54.

NAURA M., ROBINSON M., 1998. Principles of using River Habitat Survey to predict the distribution of aquatic species: an example applied to the native white-clawed crayfish Austropotamobius pallipes. Aquatic Conserv: Mar. Freshw. Ecosyst, 8, 515-527.

NEVEU A., 2000a. Étude des populations d'Austropotamobius pallipes (Crustacea, Astacidae) dans un ruisseau forestier de Normandie. I. Structure démographiques et croissance: stabilité et variété au cours de six années. Bull. Fr. Pêche Piscic., 356, 71-98.

NEVEU A., 2000b. Étude des populations d'Austropotamobius pallipes (Crustacea, Astacidae) dans un ruisseau forestier de Normandie. II. Répartition en fonction de la structure des habitats: stabilité et variabilité au cours de cinq années. Bull. Fr. Pêche Piscic., 356, 99-122.

PETERSEN R.C. JR., 1992. The RCE: a Riparian, Channel, and Environmental Inventory for small streams in the agricultural landscape. Freshwater Biology,. 27, 295-306.

RALLO A., GARCìA-ARBERAS L., 2000. Population structure and dynamics and habitat conditions of the native crayfish Austropotamobius pallipes in a pond: a case study in Basque Country (Northern Iberian Peninsula). Bull. Fr. Pêche Piscic., 356, 5-16.

RALLO A., GARCìA-ARBERAS L., 2002. Differences in abiotic water conditions between fluvial reaches and crayfish fauna in some northern rivers of the lberian Peninsula. Aquat. Liv. Res., 15, 119-128.

REYNOLDS J.D., 1979. Ecology of Austropotamobius pallipes in Ireland. Freshwater Crayfish, 4, 215-219.

REYNOLDS J.D., 1997. The present status of freshwater crayfish in Ireland. Bull. Fr. Pêche Piscic., 347, 693-700.

REYNOLDS J.M., MATTHEWS M.A., 1995. Conservation strategies for the Irish freshwater crayfish. In: REYNOLDS J.D. (ed.), The conservation of Aquatic Systems, 151-155, Royal Irish Academy, Dublin. 
ROGERS W.D., HOLDICH D.M., 1995. Survey of white-clawed crayfish distribution in the tributaries of the rivers Usk and Wye. Contract Science Report 137, Countryside Council for Wales, Bangor.

ROSCOE M.J., 1986. Distribution of the freshwater crayfish Austropotamobius pallipes in South-East Wales. Small Grant Ecological Report to the British Ecological Society, UK.

ROUSE D.B., KASTNER R.J., REDDY K.S., 1995. Toxicity of ammonia and nitrite to hatching redclaw crayfish, Cherax quadricarinatus. Freshwater Crayfish, 10, 298303.

SKURDAL J., TAUGBOL T., 2001. Crayfish of commercial importance. Astacus. In HOLDICH D.M. (ed.), Biology of freshwater crayfish. Blackwell Science, Oxford, pp. 467-510.

SLATER F.M., 2001. The demise of the native white-clawed crayfish (Austropotamobius pallipes). Breconshire Wildlife \& Naturalist, 73, 10-11.

SMITH G.R.T., LEARNER M.A., SLATER F.M., FOSTER J., 1996. Habitats features important for the conservation of the native crayfish Austropotamobius pallipes in Britain. Biol. Conserv., 75, 239-246.

SUMMERS D.W., 1996. A preliminary Investigation into the Distribution and Habitat Use of White-Clawed Crayfish (Austropotamobius pallipes) in the River Piddle. The Game Conservancy Trust, Hampshire.

THOMAS W., INGLE R., 1971. The nomenclature, bionomics and distribution of the crayfish, Austropotamobius pallipes (Lereboullet) (Crustacea, Astacidae) in the British Isles. Essex Nat., 32, 349-360.

TROSCHEL H.J., 1993. Vorkommen und Sicherung der Krebsbestände im Südwesten Baden-Württembergs. Fischereiforschungsstelle des Landes Baden-Württemberg, Langenarger, Germany, 82 p., unpubl.

TROSCHEL H.J., 1997. Distribution and ecology of Austropotamobius pallipes in Germany. Bull. Fr. Pêche Piscic., 347, 639-647.

TROSCHEL H.J., 2002. Dohlenkrebs (Austropotamobius pallipes). In: FARTMANN T., GUNNEMANN H., SALM P. und SCHRÖDER E. (2001), Berichtspflichten in Natura2000-Gebieten, Bundesamt für Naturshutz, Bd. 42, S. 417-420.

TROUILHE M.C., RICARD F., PARINET B., GRANDJEAN F., SOUTY-GROSSET C., 2003. Management of the white-clawed crayfish (Austropotamobius pallipes) in Western France: abiotic and biotic factors study. Bull. Fr. Pêche Piscic., 370-371, 97-114.

WESTMAN K., 1985. Effects of habitat modifications on freshwater crayfish. In: ALABASTER J.S. (ed.), Habitat modification on freshwater fisheries, 245-255, Butterwoths, London. 
